\title{
GOUT FLARE IN A YOUNG WOMAN WITH HYPERCATABOLIC STATE UNDER DIURETIC THERAPY
}

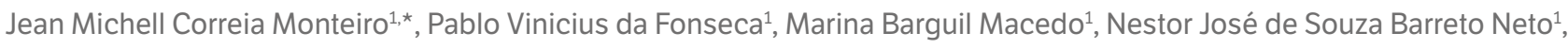 \\ Lissiane Karine Noronha Guedes ${ }^{1}$, Samuel Katsuyuki Shinjo ${ }^{1}$, Diogo Souza Domiciano ${ }^{1}$
}

1. Hospital das Clinicas HCFMUSP, Faculdade de Medicina, Universidade de São Paulo, São Paulo (SP), Brazil.

*Corresponding author: jk_michell@hotmail.com

\section{BACKGROUND}

Because female sex hormones increase urinary excretion of uric acid, premenopausal women have a substantially lower prevalence of gout compared with men (2.0\% vs. 5.9\%). Almost all the gout in females appears after menopause.

\section{CASE REPORT}

A 40-year-old woman with familiar hypertrophic cardiomyopathy and cardiac congestive insufficiency was admitted to emergency room with severe heart failure. She had been having multiple cardiac decompensations in the last year and was under chronic diuretic therapy (furosemide $160 \mathrm{mg} /$ day). Furthermore, she was submitted to electrical cardioversion for acute atrial flutter 30 days earlier. Despite optimized treatment, patient developed acute renal injury and refractory ventricular tachyarrhythmia. She was put on mechanical ventilation, intra-aortic balloon support and continuous veno-venous hemofiltration. After 11 days, her clinical status improved. After waking up from sedation, the patient started complaining of intense pain in left knee and both ankles. Of note, she reported 3-year history of recurrent episodes of inflammatory arthralgia in different joints (shoulders, ankles, left knee and small joints of hands and feet), with no definite cause. Moreover, she had previous laboratory tests that showed serum uric acid of $12 \mathrm{mg} / \mathrm{dL}$. On rheumatology examination, there were pain and warm swelling in right ankle and left knee with decreased range of motion. A high probability of gout flare was suspected. Intravenous methylprednisolone ( $20 \mathrm{mg} /$ day) was introduced to alleviate symptoms along with allopurinol $100 \mathrm{mg} /$ day, but a differential approach was performed. Laboratory workup confirmed hyperuricemia with marked reduction after continuous dialysis ( $2 \mathrm{mg} / \mathrm{dL}$ ), as well as creatinine $1.8 \mathrm{mg} / \mathrm{dL}$, with elevated C-reactive protein (CRP) $48.5 \mathrm{mg} / \mathrm{L}$ ), negative autoantibodies (rheumatoid factor, anti-CCP and anti-cell antibody), normal thyroid function and negative viral serologies. Left knee arthrocentesis was unsuccessful and joint ultrasonography demonstrated double contour cartilage line in left knee, elbows, and ankles, confirming microcrystalline arthritis (gout) as probable diagnosis, without puncturable volume of synovial fluid. At this moment, low dose colchicine ( $0.5 \mathrm{mg} /$ day) was associated. There was improvement of symptoms $2-3$ days after therapeutic institution, but she evolved with new episode of tachyarrhythmia with cardiogenic shock and death.

\section{CONCLUSION}

Albeit rare, gout in premenopausal women usually occurs with precipitating factors, especially diuretic treatment in the presence of hypercatabolic states such as congestive heart failure, renal insufficiency, and anorexia nervosa. Moreover, conditions associated with great variability of serum uric acid, such as dialysis, can promote gout flares. This case reinforces that knowledge of these risk factors frequently observed in female gout might improve outcomes in this population.

\section{KEYWORDS}

Gout, Heart failure, Hypertrophic cardiomyopathy. 